\title{
RINGS AND MODULES \\ IN HONOUR OF PATRICK F. SMITH'S 65TH BIRTHDAY
}

Website: http://pfs2008.cii.fc.ul.pt/

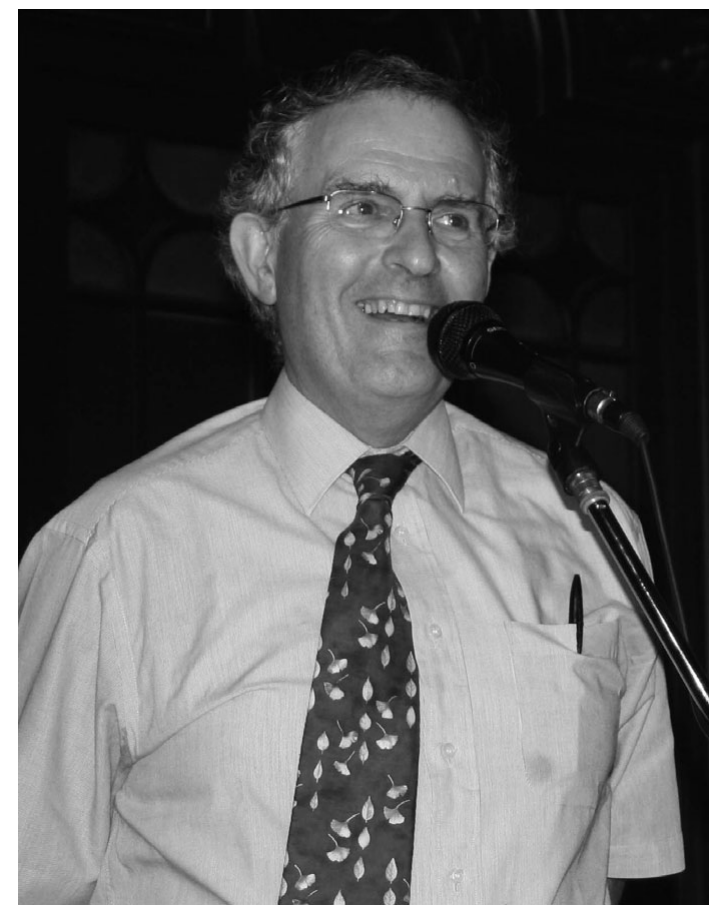

Patrick F. Smith

The conference 'Rings and Modules', in honour of Patrick F. Smith's 65th birthday and on the occasion of his retirement, gathered in Lisbon 67 of Patrick Smith's students, co-authors and friends from all over the world. The conference was held at Centro de Álgebra da Universidade de Lisboa (CAUL), 16th-19th September 2008.

The invited speakers were international experts in Ring and Module Theory and related subjects, also being long-term friends of Patrick, and talked about many aspects of rings, modules and algebras, not forgetting to mention Patrick's role in their lives, his scientific achievements and, above all, his friendship:

- Toma Albu (Bucharest, Romania), Primal, completely irreducible, and primary meet decompositions in modules;

- Alberto Facchini (Padova, Italy), Monogeny class, epigeny class, upper part, lower part;

- José Luis Gómez Pardo (Santiago de Compostela, Spain), Fitting's Lemma for non-finitely generated modules;

- Charudatta Hajarnavis (Warwick, UK) - Patrick Smith and the Artin-Rees property; 
- Christian Lomp (Porto, Portugal), Regular and biregular module algebras;

- Sergio Lopez-Permouth (Ohio, USA), Rational power series, sequential codes and periodicity of sequences;

- Barbara Osofsky (Rutgers, USA), Injective hulls of a ring with compatible ring structures;

- Mike Prest (Manchester, UK), Decomposition of pure-injective modules;

- Edmund Puczylowski (Warsaw, Poland), Linear properties of the Goldie dimension of modules and modular lattices;

- Adnan Tercan (Hacettepe, Turkey), $C_{11}$-modules;

- Robert Wisbauer (Düsseldorf, Germany), Monads and comonads in module categories.

There were also 27 contributed talks, covering a wide range of subjects (listed below). The diversity of topics is also reflected in these proceedings, and we wish to thank all the speakers and all the authors for their contributions. The Proceedings of the conference contains 14 articles, including 8 contributions from the invited speakers.

Paying tribute to Patrick could not be done without having a lot of fun and the conference dinner was a cheery event, including many speeches and a few ceilidh steps. The conference itself had finished earlier in the afternoon in the tradition of any Scottish gathering, with all the participants happily (even if not in tune...) singing Auld Lang Syne:

For auld lang syne, my dear,

for auld lang syne,

we'll tak a cup o' kindness yet,

for auld lang syne.

This conference would have not been possible without the financial support of the following funding bodies:

- Centro de Álgebra da Universidade de Lisboa (CAUL);

- Centro de Estruturas Lineares e Combinatórias (CELC);

- Centro de Matemática e Aplicações Fundamentais (CMAF);

- Edinburgh Mathematical Society (EMS);

- Fundação Calouste Gulbenkian (FCG);

- Fundação para a Ciência e a Tecnologia (FCT);

- Glasgow Mathematical Journal Research and Learning Support Fund (GMJ);

- Research Group in Algebra, University of Murcia;

- University of Glasgow, Department of Mathematics.

and the help from:

- Associação de Turismo de Lisboa (ATL);

- Banco Espírito Santo (BES);

- Câmara Municipal de Lisboa (CML);

- Complexo Interdisciplinar da Universidade de Lisboa (CIUL);

- Fundação da Universidade de Lisboa (FUL).

We gratefully acknowledge all of their contributions.

Finally I would like to thank our scientific committee,

- Ken A. Brown (Glasgow, UK);

- John Clark (Otago, New Zealand);

- José Luis Gómez Pardo (Santiago de Compostela, Spain);

- Dinh Van Huynh (Ohio, USA); 
- Barbara Osofsky (Rutgers, USA);

- Robert Wisbauer (Düsseldorf, Germany).

for their help and suggestions, as well as my co-organisers,

- Nguyen Viet Dung (Ohio, USA);

- Pedro Antonio Guil Asensio (Murcia, Spain);

- Robert J. Marsh (Leeds, UK).

and CAUL's secretary Patrícia Paraíba, for their help in organising this conference. Thanks also to the editors,

- Robert J. Marsh (Leeds, UK) (Chair);

- Ken A. Brown (Glasgow, UK);

- Pedro Antonio Guil Asensio (Murcia, Spain).

and to Ian Strachan (Glasgow, UK), chief editor of the Glasgow Mathematical Journal.

A final word of thanks to Patrick, for being such an inspiration!

Catarina Santa-Clara, local organiser, May 2010.

\section{Contributed talks}

- Pıýnar Aydoğdu, Chain conditions on non-summands;

- Adalbert Bovdi, Lie properties of crossed products;

- Victor Bovdi, Group algebras whose Lie algebras of skew-symmetric elements are Engel;

- Stelios Charalambides, Uniform modules relative to a torsion theory;

- Septimiu Crivei, On the Osofsky-Smith theorem;

- Semra Doğruöz, The relationship between torsion theories related with Goldie torsion theory and UC-modules relative to torsion theories;

- Laiachi El Kaoutit, Invertible unital bimodules over rings with local units, and related exact sequences of groups;

- Sergio Estrada, Pure injectivity with respect to cotorsion pairs;

- Marzena Filipowicz-Chomko, On rings which are filial and left but not right filial;

- Yuval Ginosar, On non-degenerate and bijective cohomology classes;

- Jairo Z. Gonçalves, Algebraic elements as free factors in simple artinian rings;

- Marcela González Peláez, On $\tau$-full modules;

- Małgorzata Hryniewicka, On PI property for reduced algebras;

- Ali Jaballah, Alternative characterizations of ring extensions satisfying some finiteness conditions;

- Surender K. Jain, Conditions as to when a ring is Noetherian;

- Fatih Karabacak, On SIP-extending modules;

- Derya Keskin Tütüncü, Mixed injective modules;

- Jan Krempa, Around uniform dimension of modules;

- Leandro Marin, AB5 in the category of firm modules;

- Jan Okniński, Finitely presented monomial algebras;

- César Polcino Milies, Lie properties of symmetric elements in group algebras;

- Francisco Raggi, Basic preradicals and main injective modules; 
- Bulent Sarac, Semiprime and radical submodules;

- Ernesto Spinelli, On the *-minimality of algebras with involution;

- Figen Tak1l, Modules whose submodules are essentially embedded in some direct summands;

- Gil Vernik, On the center and the semi-center of the enveloping algebra of a finite dimensional solvable Lie algebra over a field of prime characteristic;

- Ivan Yudin, Skew product of rings over a monoid.

\section{List of Participants \\ for the International Conference "RINGS AND MODULES"}

Albu, Toma
Arroyo, Maria José
Aydoğdu, Pınar
Bovdi, Adalbert
Bovdi, Victor
Brown, Kenneth A.
Caenepeel, Stefaan
Catino, Francesco
Charalambides, Stelios
Clark, John
Crivei, Septimiu
Doğruöz, Semra
El Kaoutit, Laiachi
Estrada, Sergio
Facchini, Alberto
Fernandez-Alonso, Rogelio
Filipowicz-Chomko, Marzena
Galvão, Maria Luísa
Garcia, José L.
Ginosar, Yuval
Gómez Pardo, José Luis
Gonçalves, Jairo
González Peláez, Marcela
González Rodrguez, Ramón
Guil Asensio, Pedro Antonio
Hajarnavis, Charudatta
Hryniewicka, Malgorzata
Jaballah, Ali
Jain, S.K.
Jespers, Eric Vrije
Karabacak, Fatih
Keskin Tütüncü, Derya
Krempa, Jan
Lobillo, Fco Javier
Lomp, Christian
Gata

Albu, Toma

Arroyo, Maria Jose

Aydoğdu, Pınar

Bovdi, Adalbert

Bovdi, Victor

Brown, Kenneth A.

Caenepeel, Stefaan

Catino, Francesco

Clark, John

Crivei, Septimiu

Doğruöz, Semra

El Kaoutit, Laiachi

Estrada, Sergio

Facchini, Alberto

Fernandez-Alonso, Rogelio

Galvão, Maria Luísa

Garcia, José L.

Ginosar, Yuval

Gómez Pardo, José Luis

Gonçalves, Jairo

González Rodrguez, Ramón

Guil Asensio, Pedro Antonio

Hajarnavis, Charudatta

Hryniewicka, Malgorzata

Jaballah, Ali

Jain, S.K.

Jespers, Eric Vrije

Karabacak, Fatih

Keskin Tütüncü, Derya

Krempa, Jan

Lomp, Christian
Romanian Academy, Romania

Universidad Autonoma Metropolitana, Mexico

Hacettepe University, Turkey

University of Debrecen, Hungary

University of Debrecen, Hungary

University of Glasgow, UK

Vrije Universiteit Brussel, Belgium

Università del Salento, Italy

Norwegian University of Science and Technology,

Norway

University of Otago, New Zealand

Babes-Bolyai University, Romania

Adnan Menderes University, Turkey

Universidad de Granada, Spain

Universidad de Murcia, Spain

Università di Padova, Italy

Universidad Autonoma Metropolitana, Mexico

Bialystok Technical University, Poland

Universidade de Lisboa, Portugal

Universidad de Murcia, Spain

University of Haifa, Israel

Universidade de Santiago de Compostela, Spain

Universidade de São Paulo, Brazil

Instituto Tecnológico Autónomo de Mexico, Mexico

Universidade de Vigo, Spain

Universidad de Murcia, Spain

University of Warwick, UK

University of Bialystok, Poland

University of Sharjah, United Arab Emirates

Ohio University, USA

Universiteit Brussel, Belgium

Anadolu University, Turkey

Hacettepe University, Turkey

University of Warsaw, Poland

Universidad de Granada, Spain

Universidade do Porto, Portugal 
Lopez-Permouth, Sergio

Macauley, Monica

Macleod, Marjorie

Marciniak, Dorota

Marin, Leandro

Matczuk, Jerzy

Miccoli, Maria Maddalena

Nogueira, Maria Teresa

Okninski, Jan

Osofsky, Barbara

Özcan, A. Çiğdem

Perone, Marco

Polcino Milies, César

Prest, Mike

Puczylowski, Edmund

Pusat-Yilmaz, Dilek

Raggi, Francisco

Rios, José

Rodriguez Gonzalez, Nieves

Santa-Clara, Catarina

Sarac, Bulent

Semião, Paulo

Siciliano, Salvatore

Simões, Maria Elisa

Simões, Raquel

Smith, Patrick F.

Spinelli, Ernesto

Tak1l, Figen

Tercan, Adnan

Vernik, Gil

Wisbauer, Robert

Yudin, Ivan
Ohio University, USA

University of Glasgow, UK

University of Glasgow, UK

Universidade Técnica de Lisboa, Portugal

Universidad de Murcia, Spain

University of Warsaw, Poland

Università del Salento, Italy

Universidade de Lisboa, Portugal

University of Warsaw, Poland

Rutgers University, USA

Hacettepe University, Turkey

Università degli studi di Padova, Italy

Universidade de São Paulo, Brazil

University of Manchester, UK

University of Warsaw, Poland

Izmir Institute of Technology, Turkey

Universidad Nacional Autonoma de Mexico, Mexico

Universidad Nacional Autonoma de Mexico, Mexico

Universidade de Santiago de Compostela, Spain

Universidade de Lisboa, Portugal

Hacettepe University, Turkey

Universidade do Algarve, Portugal

Università del Salento, Italy

Universidade de Lisboa, Portugal

University of Leeds, UK

University of Glasgow, UK

Università del Salento, Italy

Anadolu University, Turkey

Hacettepe University, Turkey

Haifa University, Israel

Heinrich-Heine-Universität, Germany

Universidade de Coimbra, Portugal 